
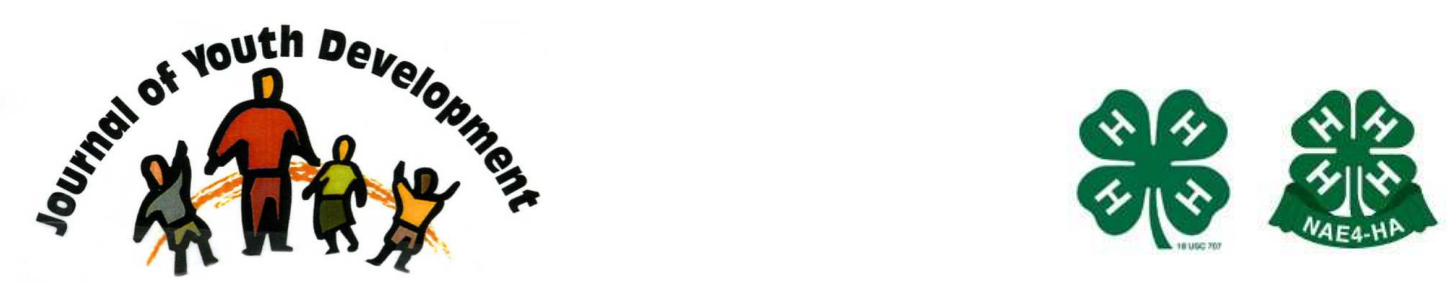

Bridging Research \& Practice

\title{
Character Development through Youth Sport: High School Coaches' Perspectives about a Character-based Education Program
}

\author{
Kaitlyn A. Ferris \\ Tufts University \\ kaitlyn.ferris@tufts.edu \\ Andrea Vest Ettekal \\ Tufts University \\ Jennifer P. Agans \\ Cornell University \\ Brian M. Burkhard \\ Tufts University
}




\title{
JOURNAL OF YOUTH DEVELOPMENT \\ bridging research and practice

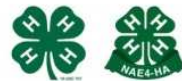

Volume 10, Number 3, Special Issue 2015

Article 151003FA010

\section{Character Development Through Youth Sport: High School Coaches' Perspectives about a Character-based Education Program}

\author{
Kaitlyn A. Ferris and Andrea Vest Ettekal \\ Tufts University \\ Jennifer P. Agans \\ Cornell University \\ Brian M. Burkhard \\ Tufts University
}

\begin{abstract}
This study examined high school sports coaches' perspectives about a character-based coach education workshop designed to promote positive coaching practices and transform the culture of youth sports. Fifteen coaches $\left(M_{a g e}=42.07, S D=14.62\right.$, $73.3 \%$ male) provided feedback about Positive Coaching Alliance's (PCA) "Double-Goal Coach" training program and what aspects of the workshop they applied to their coaching practices. Results indicated that coaches believed that participation in PCA workshops contributed to the value coaches attributed to individuals, to coachoriented character development, and to positive relationships within youth sports. The coaches also suggested changes in future PCA workshops. These findings provide preliminary evidence that coaches' incorporate skills acquired through participation in character-based coach education programs. We discuss implications for coaches and athletes, and for policies aimed at enhancing positive youth attributes developed through sport.
\end{abstract}

\section{Introduction}

Sport participation is highly prevalent in the U.S., with more than $70 \%$ of adolescents participating in at least one sport (Sabo \& Veliz, 2008). Widespread engagement in sports during the after-school hours suggests that sports have the potential to reach, and possibly positively impact, over 20 million youth (National Sport Goods Association, 2011). However, 
there are mixed findings regarding the impact of sports on youth development. On the one hand, because sports are skill-focused and offer opportunities for challenge, participation in sports is related to increased initiative, time management skills, goal pursuit, and persistence (Holt \& Neely, 2011). On the other hand, the "win at all cost" sport mentality (Shields, Bredemeier, LaVoi, \& Power, 2005) leads some athletes to feel increased stress, often due to the extreme focus on achievement and public "tests" of athletes' abilities (Scanlan, Babkes, \& Scanlon, 2005) or competition among teammates for playing time (Brustad, Babkes, \& Smith, 2001).

Coaching behaviors have explained some of the variation in the outcomes associated with sport participation (Coté, Bruner, Erickson, Strachan, \& Fraser-Thomas, 2010). In recent years, youth sport coaches have come to mirror professional sports coaches, shifting the focus from sport as a leisure setting, where youth have fun, to a focus on winning (Fraser-Thomas, Coté, \& Deakin, 2005). Young athletes are highly influenced by their sport coaches and look to their coaches for guidance on and off the playing field (Coté et al., 2010). Playing for a coach whose philosophy is grounded solely in winning may contribute to negative outcomes among today's athletes (Farrey, 2009). For example, youth who had controlling and autocratic coaches reported less enjoyment and eventually dropped out of sport (Pelletier et al., 2001), whereas players having encouraging, supportive, and democratic coaches had positive, character-building sport experiences, such as reduced cheating and improved sportsmanship (Doty, 2006). Effective coach education programs help train coaches to promote a balance between athletes' scoreboard and life success (Cushion, Armour, \& Jones, 2003), resulting in long-term positive outcomes, including improved character beyond the sport setting (Holt \& Neely, 2011).

Taken together, these findings highlight the considerable impact of coaches on athletes' development and call attention to the need for formal training to teach the coaches of youth sports to adopt positive, character-building coaching strategies (Falcao et al., 2012). In this study, we examined one of the largest coach education programs across the U.S., a researchbased curriculum developed by the national non-profit organization, Positive Coaching Alliance (PCA). PCA has reached many coaches (i.e., 113,333 coaches in 2014 alone; T. Syer, personal communication, August 26, 2015) since its inception in 1998, partnering with several national youth sport organizations (e.g., U.S. Lacrosse) and school districts across the U.S. PCA's mission for coaches is to train them to be "Double-Goal Coaches" or to emphasize winning (i.e., Goal 1), as well as life-skills development (i.e., Goal 2). Testimonials about PCA from professional athletes and alumni of the program speak to its positive impact on athletes' character development; however, empirical evidence is needed to demonstrate PCA's effectiveness. The goal of this article is to provide initial insights about coaches' perspectives about this characterbased coach education program.

\section{The Role of Coaches in Athletes' Character Development}

According to relational developmental systems metatheory (Overton, 2015), development is the result of bidirectional relations between youth and their environments (represented as individual $\leftarrow \rightarrow$ context relations). Lerner and Callina (2014) extended this framework to the study of character development, highlighting the importance of individual $\leftarrow \rightarrow$ individual relations, in addition to individual $\leftarrow \rightarrow$ context relations, for character outcomes. From the positive youth development perspective (PYD; Lerner et al., 2015), development is optimized when individual $\leftarrow \rightarrow$ individual, or individual $\leftarrow \rightarrow$ context, relations are mutually beneficial. As a result, athletes' development in youth sports may be strongly influenced by interactions with coaches (Cushion et al., 2003). Several notable conceptual frameworks about sport coaching also align with these theoretical ideas, suggesting that coaching is both an individual and social 
process (for review, see Coté et al., 2010). Moreover, youth developmental outcomes are optimized in the context of positive, sustained relationships with adult leaders, such as coaches (Lerner et al., 2015).

Although most coaches have the ability to teach sport-specific skills (Holt \& Neely, 2011), many coaches have limited access to resources and training specifically related to character development or athlete mentorship (Falcao et al., 2012). As such, it would be useful to capitalize on "teachable moments" that foster character development (Coakley, 2011). PCA is one of the largest coach education programs in the U.S. It explicitly focuses on positive individual and interpersonal goals (i.e., character development), and therefore, may have the potential to provide coaches with the skills needed to create a sport culture that promotes character. However, consistent with the theory of planned behavior (Ajzen, 2012), beliefs about behaviors predict individuals' behavioral intentions and actual behaviors. Therefore, in order for PCA to foster character-promoting coaching behaviors, coaches must first believe in the importance and value of such behaviors. Examining coaches' beliefs and behaviors following participation in PCA programming will provide greater insight into workshop effectiveness.

In the current study, we examined whether coaches' valued PCA principles learned through participation in the PCA program, as well as their behavioral intentions. Not only does PCA have a commitment to changing youth sport culture by changing coaches' behaviors, the organization has a growth mindset (Thompson, 2010). That is, PCA is openly receptive to feedback from program participants and partner organizations to enhance the content, structure, and delivery of the program's curriculum. To this end, we also examined coaches' feedback about the workshop, in that such opinions might be used to improve the curriculum.

\section{Study Goals}

As a first step toward understanding PCA's "Double-Goal Coach" program, we examined coaches' perspectives about PCA training in regard to: 1 . The value of the program, 2 . Their intention to change their behaviors following participation in the program, and 3 . Their feedback about potential improvements to the program.

\section{Method}

Data for this study came from a larger, mixed-methods study evaluating the PCA model (Ettekal et al., 2015). At this writing, the project is ongoing, and assesses coaches and athletes in the greater Boston area for three years. Four schools that vary in regard to socioeconomic status and ethnic composition are included, following a waitlist control design for PCA program delivery. The major focus of the project involves evaluating athlete outcomes after receiving PCA programming. As such, very little data were collected from coaches. However, all coaches receiving PCA programming were asked to complete surveys answering questions specific to their PCA training.

Data included in this study are from the first year of the project and involve only those coaches in the two schools receiving PCA programming who provided responses to PCA-specific survey questions $(N=15)^{1}$. The sample was comprised of coaches $\left(M_{a g e}=42.07, S D=14.62\right)$ who were mostly male (73.3\%) and White (70\%). The majority of coaches (90.9\%) had at least a

\footnotetext{
${ }^{1}$ In total, 65 coaches were enrolled in the first year of the study. We eliminated 20 coaches who were not in schools receiving PCA programming, and 30 coaches who were in schools receiving PCA programming, but who did not respond to the PCA-specific questions on the survey.
} 
bachelor's degree, and $72.7 \%$ had at least five years of prior coaching experience. Coaches from a variety of sports (e.g., soccer, basketball, tennis) across fall, winter, and spring seasons were represented.

\section{Procedures}

At the beginning of each athletic season, coaches were invited to receive PCA's "Double-Goal Coach: Coaching for Winning and Life Lessons" training program (Thompson, 2010). Self-report surveys were administered to coaches before the beginning of the PCA training program and at the end of the season. First, we describe the implementation of the PCA program, and then we provide details about the surveys administered to coaches.

Positive Coaching Alliance programming. PCA training involves a one- to two-hour, interactive workshop at the beginning of the sport season. The workshop features information from expert coaches and sport psychologists, as well as interactive discussions of scenarios coaches and athletes may encounter throughout the athletic season. All sessions are led by "trainers" who are former coaches and athletes themselves, and who receive in-depth training on content and delivery style. The content of the curriculum focuses on two goals of coaching: 1. Scoreboard success, or mastery of sport-specific skills, and 2. Life lessons, or character development. As many coaches have the training to teach sport-specific skills (Holt \& Neely, 2011), PCA training is centered on the latter goal.

PCA trains coaches to transform athletes into "competitors of character," teaching life lessons in three domains: 1. Self; 2. Team; and 3. Game (Thompson, 2010). Trainers provide corresponding "tools" for each domain to help coaches foster athletes' character development. For example, PCA uses the "ELM tree of mastery" (corresponding to "self" outcomes), which emphasizes athletes maximizing their Effort, focusing on Learning and personal improvement, and bouncing back from Mistakes). An example of a PCA tool reinforcing team outcomes is "emotional tank-filling." PCA suggests that coaches should fill their athletes' emotional tanks by following a 5:1 ratio of positive appraisals to constructive criticisms. PCA uses the acronym "ROOTS" to promote athletes' contributions to a better game, such that they should respect the

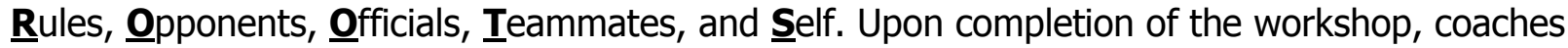
are certified as a PCA "Double-Goal Coach," and are encouraged to implement these tools in practices and games throughout their season through the use of supplemental materials, such as books detailing the core PCA principles (Thompson, 2010), and weekly talking points for coaches to use with athletes.

Self-report surveys. At the end of the season, coaches completed self-report surveys either in-person or online. In this study, we used coaches' responses to five open-ended questions to assess if coaches valued the information presented at the workshop, intended to change their behaviors, and had comments about the information presented in the workshops. The questions were:

1. What was the most valuable thing you learned in your PCA training?

2. Please describe what you did not like or found irrelevant about the PCA training.

3. Please describe what you did differently based on your PCA training, if anything.

4. Please describe how your athletes applied or did not apply the PCA tools.

5. Is there anything else you'd like to say about your PCA training?

\section{Analytic Strategy}

We used inductive and deductive qualitative content analysis in NVivo 10 (Bazeley \& Jackson, 2013). Content analysis is a useful method for identifying and categorizing important pieces of 
text in order to make inferences about the data. Coding began with a directed content coding analysis. First, two authors read coaches' written responses and took notes about three specific themes, developed from existing theory and empirical research (Lerner \& Callina, 2014; Shields et al., 2005). These themes involved perspectives about coaching discussed in the workshop: 1. coach-oriented (i.e., individual); 2. coach-athlete-oriented (i.e., relational); and 3. characterbased education program-oriented (i.e., environmental). Coding was an iterative process using investigator triangulation to enhance validity (Farmer, Robinson, Elliot, \& Eyles, 2006). Coders double-coded all of coaches' written responses, discussing any discrepancies, and continually updated the coding manual until adequate inter-rater reliability was achieved (kappa $=.86$; Gwet, 2014). After the first round of coding, a conventional content coding analysis (i.e., inductive approach) was used to identify sub-themes within each of the three broad themes. That is, the authors read all responses for a given theme and then worked collaboratively to identify emergent sub-themes (DeCuir-Gunby, Marshall, \& McCulloch, 2011).

\section{Results}

Evidence for the three overarching themes was found in coaches' responses. First, coaches discussed coach-oriented perspectives, which focused on the growth and development of their coaching behaviors as a result of participating in PCA's "Double-Goal Coach" workshop (mentioned 38 times by 15 coaches). Coaches also emphasized the importance of athletes' development, and the role of relationships in athletes' character development (mentioned 19 times by 10 coaches). Finally, coaches' perspectives included feedback about how PCA workshops could be improved for future attendees (mentioned 28 times by 10 coaches).

Taken together, the pattern of frequencies in which the coded themes were mentioned is not surprising given that the content of the "Double-Goal Coach" workshop aims to strike a balance between providing coaches with the necessary tools (i.e., ELM tree of mastery, emotional tankfilling, ROOTS) to transform athletes into "competitors of character" and, as well, opportunities to reflect on their own coaching strategies. In the next sections, we use excerpts of coaches' responses to illustrate a more detailed discussion of each theme. The findings within each theme are organized by the sub-themes that emerged. Pseudonyms are used throughout the results to protect participants' identities.

\section{Coach-oriented Perspectives}

Two sub-themes emerged in coaches' discussions surrounding coach-oriented perspectives (mentioned by 15 coaches), namely self-reflection and the intent to change. First, several coaches expressed self-reflection by tying the PCA training back to their current coaching behaviors. For example, Maria, who coaches track and field, said "[PCA] just made me more cognizant of my own [coaching] practices." Similarly, Patrick, who coaches boys' basketball, said he was "more aware" of his coaching behaviors after PCA training. Patrick tied his reflection back to his coaching philosophy stating, "PCA training really helped solidify my beliefs as a coach. I have always talked about life lessons and bigger picture issues. After having the PCA training, it made me confident in what I was teaching." In a similar vein, David, a track and field coach, talked about the importance of continued learning, even after having coached for 31 years. David said, "I still read the rule book every year. I attend clinics each year. I read journals, articles, and books on the sport, and update my training methods. I still know that I can learn something new each season." These perspectives suggest that PCA training encouraged coaches to critically consider their coaching strategies. 
Next, coaches discussed their intentions to change their coaching behaviors after the PCA workshop. Many of the coaches thought the PCA training provided important information, such as Paul who said, "there were many valuable issues covered in the formal presentation. Going over the elements of what makes a positive athletic experience was very helpful." In turn, these valuable insights prompted many coaches to change their current coaching behaviors. The most frequent way that coaches described changing was by becoming more "constructive," "staying positive," and offering "continued praise." For example, Ann said that she now "keep[s] things positive, even after losing a game." Peter stated this idea most clearly saying that it is important to:

Instill positive mentality to athletes about competing and supporting each other, on and off the field, and to establish a solid culture of dedication and encouragement for all levels, in order to value the process (and product) of 'winning' [sic] and improving.

Taken together, these statements suggest that coaches go beyond critically considering their own coaching behaviors, and report that they take actions to change such behaviors towards fostering a character-building sport culture.

\section{Relationships in the Sports Context}

As illustrated in the previous section, some coaches' perspectives of the PCA training focused on their individual development. Coaches also discussed their perspectives about the role of athletes following PCA training (mentioned by 10 coaches). Two sub-themes emerged in coaches' discussions of athletes, namely the outcomes that athletes should exhibit and positive relationships in sport settings. First, coaches discussed the importance of developing the "whole" athlete. For example, Arthur, a girls' basketball coach, said the most valuable thing he learned from the PCA training was to "focus on building individuals, not just wins and losses." Similarly, Steven, a boys' soccer and tennis coach, saw his athletes extending what they learned in sport to other contexts, such as "working hard in class." Mary discussed the importance of building athletes' character:

A lot about being a good student athlete comes down to character. The right character will prevail not only on the field, but in every other institution. Character is everything, and possessing integrity that is morally sound will lead to a more successful life.

Overall, these coaches described the importance of athletes being well-rounded individuals, focusing not only on the game, but on the broader character attributes fundamental to engaged citizenship.

Coaches also discussed how they saw sport-specific relationships change following the PCA training. Some of these discussions centered on the coach-athlete relationship. For example, Chris, who coaches' football and girls' basketball, said, "The relationships you develop with your athletes are very important." Similarly, Catherine said "we are mentors, as well as coaches." Other coaches saw improvements in their athletes' relationships with fellow teammates. David had "a great feeling of team, with stronger performers helping new or younger athletes as much as focusing on their own improvement." Two coaches pointed specifically to change among the team captains. For example, Mary said, "The captains took PCA training to heart and did a great job holding their teammates accountable for their actions on the track and off. They did a great job helping motivate younger athletes towards improving their skill and work ethic." Similarly, Ann witnessed her "athletes [being] nicer to each other and captains [taking] their leadership role more seriously." These perspectives suggest that training coaches to take on the responsibility of winning, as well as promoting life skills, can potentially positively impact relationship outcomes within youth sports settings. 


\section{Constructive Feedback and Workshop Improvements}

Coaches' perspectives also reflected specific feedback about the "Double-Goal Coach" workshop (mentioned by 10 coaches). In particular, coaches' gave feedback in regard to the structure, as well as the content of the workshop. Most of the responses regarding the structure of the PCA training addressed the logistics of implementing the program. On the one hand, some coaches thought the workshop (which lasted between one to two hours) was too long. For instance, male football coach, Michael, said, "It seemed longer than needed. Two shorter sessions would have been greater than one long one." On the other hand, other coaches, specifically Ann, believed "[PCA training] was a little short," and would have preferred a longer workshop. David was also burdened by the timing because, "trying to find the right time to do the meetings is a difficult proposition with all the commitments that our athletes and coaches have with school, work, and family." Similarly, Paul felt "the timing of the event would have been less imposing upon the coaching staff if the training could have been arranged to meet before the official start of the season."

Nevertheless, other coaches liked the training enough to think that "an annual training session of this program would be very much beneficial" and wanted their school to continue the partnership with PCA in subsequent years. These coaches' perspectives show variation in views regarding the length of workshops and, in addition, highlight the various roles that sports coaches and athletes assume and the importance of considering their numerous non-sport responsibilities when designing PCA programming.

Coaches also commented on the content of the workshop. Overall, most coaches thought the content was very informative, such as one coach who said he "loved the PCA training." Moreover, coaches thought the information was valuable, or more specifically that, "no information was irrelevant to being a better coach."

Other coaches commented about how the content was delivered. David thought that the trainers (who delivered the curriculum) "went out of their way to make the training meetings informative, enjoyable, and something athletes will carry with them." However, some coaches preferred that the content be tailored to sport-specific issues arising at their school. For example, Michael said the workshop was "very cookie-cutter," and that "our problems are not as general as other schools." Coaches also thought the workshops should be more interactive because "there was not much interaction with other coaches." This point was reinforced by Thomas, a football coach, who valued opportunities for "coaches from all sports to share common experiences and different ways of handling them." Finally, coaches wanted some athletes to participate in the workshop alongside their coaches. Catherine said, "The coaches could attend the training with the talented athletes to get a better idea of what the athletes are thinking about the program."

These responses indicate that coaches may prefer a workshop that cuts across the individuals involved in sports (e.g., coaches, athletes, officials) and is delivered in an interesting and interactive format, perhaps tailored for the specific sport.

\section{Discussion}

Coaches are influential figures in athletes' development within sports, and skills and values acquired through positive coach-athlete relationships may impact the transference of life skills across various contexts (e.g., schools, communities) of youth's lives (Fraser-Thomas et al., 
2005). Research investigating coaches' developmental outcomes is becoming increasingly necessary, given that the majority of studies examining character development in youth sports contexts have largely focused on athlete outcomes. PCA represents one of the largest character-based coach education programs in the U.S. However, in order for PCA's "DoubleGoal Coach" workshop to help coaches emphasize scoreboard and life success as part of their coaching practices, more understanding is needed about coaches' perspectives about such character-based coaching programs.

Shaping Coaches' Character through Continued Personal Growth and Development Although it is possible, character development through sport participation may not often happen by chance. Rather, the potential for character enhancement may increase when coaches make a conscious effort to create an athletic environment fostering the acquisition of moral and social values (Shields et al., 2005). Character development may be optimized when coaches align their coaching practices with ideals of sportsmanship, personal and social responsibility, positivity, and fair play (Alberts, 2003). In the current study, coaches' responses to five openended questions suggested that reflecting on one's coaching practices and reinforcing existing coaching techniques were central lessons learned through PCA training. These perspectives suggest that coaches were open to personal improvement and recognized the role of their own character in creating a sports environment where they could teach athletes sport-specific skills and life skills.

These findings suggest, then, that it may be useful to structure PCA workshops to support such coach orientations. The workshops might be structured to foster a positive learning environment to help coaches transform their current coaching philosophy into tangible coaching behaviors that foster athletes' character development in the athletic context and beyond (Fraser-Thomas et al., 2005).

Promoting Athletes' Character Development through Positive Relationships in Sport Coaches' perspectives highlight that positive relationships bolster character development (Erickson, Coté, Hollenstein, \& Deakin, 2011). These responses support the idea that character development is the result of mutually-beneficial, individual $\leftarrow \rightarrow$ context relations (Lerner \& Callina, 2014). More specifically, in the context of youth sports, character development may be optimized by positive coach $\leftarrow \rightarrow$ athlete or athlete $\leftarrow \rightarrow$ athlete interactions. Coaches' responses also highlight that mentorship, as a means to potentially promote character attributes, is a complex process involving individual and social processes (Coté et al., 2010).

The "Double-Goal Coach" program may provide a foundation of knowledge for coaches to use when acting to promote such processes. However, the effects of positive coach $\leftarrow \rightarrow$ athlete relationships may be even more long-lasting if PCA workshops provide coaches with opportunities to practice additional skills (e.g., perspective taking, empathy). Such additional skills may allow coaches to be effective sport and life coaches.

Importantly, coaches discussed how positive relationships within sports are not limited solely to coach-athlete interactions, but extend to athlete-athlete relationships. Coaches discussed how certain athletes, typically veteran players (e.g., team captains), served as role models for younger teammates. In youth sports, a positive peer motivational climate, such as one characterized by encouragement rather than competition, is conducive to positive teammate relationships (MacDonald, Coté, Eys, \& Deakin, 2011). In turn, positive peer relationships contribute to athletes' sports motivation, commitment, enjoyment, and performance (for review, see Brustad, Babkes, \& Smith, 2001). Thus, athletes' character development through sport 
participation is tied to individual- as well as team-level experiences (Duda \& Balaguer, 2007). High athletic performance and team cohesion is not possible without coaches who intentionally structure a positive team environment (Garcia-Calvo et al., 2014).

\section{Constructive Feedback and Workshop Improvements}

Coaches completing PCA's "Double-Goal Coach" workshop leave the training session certified as "Double-Goal Coaches." However, to ensure the long-term effectiveness of PCA programming, it is important that PCA trainers consider coaches' feedback and suggestions for program improvements. Attention to such feedback may improve the structure and content of the workshop. Similar to previous work (Koh, Ong, \& Camire, 2014), coaches in the current study indicated time constraints to be one of the biggest challenges to workshop effectiveness. Results from mentorship programs implemented with underserved youth in urban school settings (Martinek, Schilling, \& Johnson, 2001), indicate that exposure to the intervention program on multiple occasions may be necessary for the intervention to be effective. If such a process is occurring with coaches attending the "Double-Goal Coach" workshop for the first time, then greater exposure to the workshop curriculum over multiple training sessions may be required to observe changes in coaching practices.

Coaches' perspectives on the structure of the workshop suggested that more individualized programming may be necessary. For instance, coaches discussed scheduling conflicts often associated with coordinating a workshop that could be attended by coaches across all sports. PCA trainers may want to provide coaches with multiple times for participating in the "DoubleGoal Coach" workshop. Workshops could be held separately based on sport type, such as one workshop for football coaches and one workshop for soccer coaches, rather than one workshop for all coaches of fall sports. If this approach was taken, there could also be flexibility in the location of workshops, with workshops held in locations that coaches already frequent (e.g., their respective practice fields or courts). Multiple workshop options could reduce what coaches considered interruptions to their daily-practice routines.

Furthermore, given that online education programs are a recent and popular trend, PCA trainers could offer the "Double-Goal Coach" workshop in an online, multi-media format, which would allow coaches to complete the training based on their individual availability. Previous research (U.S. Department of Education, 2009) supports the effectiveness of online courses for older learners and youth in grades $\mathrm{K}$ through 12 . However, more research investigating the impact of online coaching modules is needed.

Finally, several aspects of coaches' perspectives suggest that smaller workshops may be valuable (Rocca, 2010). First, coaches valued the opportunity for group discussions, which are more feasible in small groups. Indeed, group discussions increase retention of material, but should be carefully monitored as they may be unproductive in some cases. For instance, group discussions could reduce the time available to cover necessary workshop content or derail the flow of the presentation. Second, in smaller groups coaches may feel less anxious about sharing their perspectives. With smaller groups, the spatial arrangement of the workshop can also be organized to facilitate conversation among attendees (e.g., arranging chairs in a circle versus rows). Finally, smaller groups would allow the trainers to tailor the workshop to the specific sport, thereby enhancing coaches' ability to relate to the content. In such small group settings, coaches could discuss different ways to handle difficult situations that commonly arise in their specific sports. Overall, these findings suggest multiple ways that the "Double-Goal Coach" workshop could be structured to optimize coaches' experiences. 


\section{Limitations and Directions for Future Research}

This article reports preliminary findings from a larger ongoing longitudinal study and must be considered in light of several limitations (Ettekal et al., 2015). First, coaches' responses were retrospective, asking them to reflect back on how they applied PCA principles throughout the season. Coaches are likely to change their coaching practices at certain points in the season, such as before important competitions (Erickson et al., 2011). Other methods, such as the Experience Sampling Method (Csikszentmihalyi \& Larson, 1987), may better capture changes in coaches' behaviors at distinct points across the athletic season. Second, changes in coaches' behaviors were documented through self-report surveys, which are subject to social desirability bias (Bowling, 2005). Multi-method approaches, such as using self-report surveys and observational methods, would yield more valid inferences about coaches' behaviors. Third, coaches were the sole reporters about their behaviors. Allowing multiple persons to rate the coaches would help determine if coaches' self-reported behaviors align with, for example, athletes' perceptions of their behaviors. Taken together, these limitations could be addressed in future research through multitrait-multimethod approaches (cf., Ferris et al., 2015) that enhance reliability and validity of data.

In addition, although the demographics of our sample are consistent with average coach characteristics across the U.S. (Shields et al., 2005), future research is needed to generalize these findings to more diverse coaching samples. Recent research indicates that characterrelated discussions often arise between ethnic minority leaders and program participants (Ferris et al., 2015); however, it is unclear whether ethnic minority coaches also approach character development with a similar coach- and relationship-oriented focus, or would identify the same areas requiring improvement in future PCA workshops.

Furthermore, more scholarship is needed contrasting the character-based focus of coaches working with youth of varying ability levels (i.e., freshmen, junior varsity, varsity teams; intramural, youth leagues). Previous research reports that coaches' approaches to working with athletes of different skill levels are varied and dependent on what coaches believe is needed to create an optimal learning environment for their athletes (Cregan, Bloom, \& Reid, 2007). To pursue these lines of future research, the need for longitudinal data among diverse samples of coaches is underscored.

\section{Conclusions}

Athletes typically have high social status in schools, and sporting events often serve to unite community members. The impact of youth athletes highlight the need to develop character attributes among these young people. Thus, PCA's motto of building "better athletes, better people" pertains to a responsibility of all youth coaches. Many youth sports programs share an underlying focus on teaching life lessons through sport (Berlin et al., 2007); however, the findings from this study emphasize the significance of gaining an understanding of coaches' perspectives about character-based education programs, and specifically about the information they value and apply in their coaching practices.

Only after scholars have an understanding of the process by which coaches integrate characterbased program curriculum into their coaching practices and daily interaction with athletes, can appropriate examination be conducted of sports as holistic contexts where coaches' and athletes' character strengths can align to transform youth sports culture into a character "development zone." Given the potential positive impact of participation in youth sports on the development of character attributes, it is important for researchers to continue to examine the 
processes through which coaches' character strengths are enhanced through participation in character-based coach education programs.

\section{References}

Ajzen, I. (2012). The theory of planned behavior. In P.A.M. Van Lange, A.W. Kruglanski, \& E. T. Higgins (Eds.), Handbook of Theories of Social Psychology: Volume One (pp. 438-459). Thousand Oaks, CA: Sage Publications Inc.

Alberts, C. (2003). Coaching issues and dilemmas: Character building through sport participation. Reston, VA: NASPE Publications.

Bazeley, P., \& Jackson, K. (Eds.). (2013). Qualitative data analysis with NVivo. Thousand Oaks, CA: Sage Publications.

Berlin, R. A., Dworkin, A., Eames, N., Menconi, A., \& Perkins, D. F. (2007). Examples of sportsbased youth development programs. New directions for youth development, 115, 85-106.

Bowling, A. (2005). Mode of questionnaire administration can have serious effects on data quality. Journal of Public Health, 2オ3), 281-291.

Brustad, R.J., Babkes, M., \& Smith, A. (2001). Youth in sport: Psychological considerations. In R.N. Singer, H.A. Hausenblas, \& C.M. Janelle (Eds.), Handbook of spork psychology (2 ${ }^{\text {nd }}$ ed., pp. 604-635). New York, NY: Wiley.

Coakley, J. (2011). Youth sports: What counts as "positive development?" Journal of Sport and Social Issues, 35(3), 306-324.

Coté, J., Bruner, M., Erickson, K., Strachan, L., \& Fraser-Thomas, J. (2010). Athlete development and coaching. In J. Lyle \& C. Cushion (Eds.), Sports coaching: Professionalisation and practice (pp. 63-84). London, England: Churchill Livingston.

Cregan, K., Bloom, G.A., \& Reid, G. (2007). Career evolution and knowledge of elite coaches of swimmers with a physical disability. Research Quarterly for Exercise and Sport, 78(4), 339-350.

Csikszentmihalyi, M., \& Larson, R. (1987). Validity and reliability of the experience-sampling method. The Journal of Nervous and Mental Disease, 175(9), 526-536.

Cushion, C.J., Armour, K.M., \& Jones, R.L. (2003). Coach education and continuing professional development: Experience and learning to coach, Quest, 55(3), 215-230.

DeCuir-Gunby, J. T., Marshall, P. L., \& McCulloch, A. W. (2011). Developing and using a codebook for the analysis of interview data: An example from a professional development research project. Field Methods, 23, $136-155$.

Doty, J. (2006). Sports build character?! Journal of College and Character, ス3), 1-9.

Duda, J.L., \& Balaguer, I. (2007). Coach-created motivational climate. In S. Jowett, \& D. Lavalle (Eds.), Social psychology in sport (pp. 117-130). Chicago, IL: Human Kinetics Inc. 
Erickson, K., Coté, J., Hollenstein, \& Deakin, J. (2011). Examining coach-athlete interactions using state space grids: An observational analysis in competitive youth sport. Psychology of Sport and Exercise, 12, 645-654.

Ettekal, A.V., Lerner, R.M., Ferris, K.A., Gansert, P.K., Agans, J.P., \& Burkhard, B.M. (2015). "Because that's where the kids are": Applying developmental science to understand the role of sport participation in positive youth development. Manuscript submitted for review.

Falcao, W.R., Bloom, G.A., \& Gilbert, W.D. (2012). Coaches' perceptions of a coach training program designed to promote youth developmental outcomes. Journal of Applied Sport Psychology, 24(4), 429-444.

Farmer, T., Robinson, K., Elliott, S.J., \& Eyles, J. (2006). Developing and implementing a triangulation protocol for qualitative health research. Qualitative Health Research, 16(3), 377394.

Farrey, T. (2009). Game on: How the pressure to win at all costs endangers youth sports, and what parents can do about it. ESPN.

Ferris, K.A., Hershberg, R.M., Su, S., Wang, J., \& Lerner, R.L. (2015). Character development Among youth of color from low-SES backgrounds: An examination of Boy Scouts of America's ScoutReach program. Unpublished manuscript submitted for review.

Fraser-Thomas, J.L., Coté, J., \& Deakin, J. (2005). Youth sport programs: An avenue to foster positive youth development. Physical Education and Sport Psychology, 10(1), 19-40.

Garcia-Calvo, T., Leo, F.M., Gonzalez-Ponce, I., Sanchez-Miguel, P.A., Mouratidis, A., \& Ntoumanis, N. (2014). Perceived coach-created and peer-created motivational climates and their associations with team cohesion and athlete satisfaction: Evidence from a longitudinal study. Journal of Sport Sciences, 1-13.

Gwet, K. L. (2014). Handbook of inter-rater reliability: The definitive guide to measuring the extent of agreement among raters. Gaithersburg, MD: Advanced Analytics, LLC.

Holt, N.L., \& Neely, K.C. (2011). Positive youth development through sport: A review. Revista iberoamericana de psicologia del ejercicio y el deporte, 6(2), 299-316.

Koh, K.T., Ong, S.W., \& Camire, M. (2014). Implementation of a values training program in physical education and sport: Perspectives from teachers, coaches, students, and athletes. Physical Education and Sport Pedagogy, 1-18.

Lerner, R.M., \& Callina, K.S. (2014). Relational developmental systems theories and the ecological validity of experimental designs. Human Development, 56(6), 372-380.

Lerner, R.M., Lerner, J.V., Bowers, E., \& Geldhof, G.J. (2015).Positive youth development and relational developmental systems. In W.F. Overton \& P.C. Molenaar (Eds.), Theory and Method. Volume 1 of the Handbook of Child Psychology and Developmental Science ( $7^{\text {th }}$ ed.). (pp. 607651) Editor-in-chief: R.M. Lerner. Hoboken, NJ: Wiley. 
MacDonald, D.J., Coté, J., Eys, M., Deakin, J. (2011). The role of enjoyment and motivational climate in relation to the personal development of team sport athletes. The Sport Psychologist, $25,32-46$.

Martinek, T., Schilling, T., \& Johnson, D. (2001). Transferring personal and social responsibility of underserved youth to the classroom. The Urban Review, 33(1), 29-45.

National Sporting Goods Association. (2011). 2011 versus 2001 youth sports participation, NSGA. Retrieved from http://www.nsga.org/files/public/2011vs2001_youth_participation_ website. pdf

Overton, W.F. (2015). Process and relational developmental systems. In W.F. Overton \& P.C. Molenaar (Eds.), Handbook of child psychology and developmental science. Vol. 1: Theory and method (7th ed., pp. 9-62). Editor-in-chief: R.M. Lerner. Hoboken, NJ: Wiley.

Pelletier, L.G., Fortier, M.S., Vallerand, R.J., \& Briere, N.M. (2001). Associations between perceived autonomy support, forms of self-regulation, and persistence: A prospective study. Motivation and Emotion, 4, 279-306.

Rocca, K.A. (2010). Student participation in the college classroom: An extended multidisciplinary literature review. Communication Education, 59(2), 185-213.

Sabo, D., \& Veliz, P. (2008). Go out and play: Youth sports in America. East Meadow, NY: Women's Sports Foundation.

Scanlan, T.K., Babkes, M.L., \& Scanlan, L.A. (2005). Participation in sport: A developmental glimpse at emotion. In J.L. Mahoney, R.W. Larson, \& J.S. Eccles (Eds.), Organized activities as contexts of development. Hillsdale, NJ: Lawrence Erlbaum.

Shields, D.L., Bredemeier, B.L., LaVoi, N.M., \& Power, F.C. (2005). The sport behavior of youth, parents, and coaches: The good, the bad, and the ugly. Journal of Research in Character Education, 3(1), 43-59.

Thompson, J. (2010). Developing winners in sports and life: The power of double-goal coaching. Portola Valley, CA: Balance Sports Publishing, LLC.

U.S. Department of Education. (2009). Evaluation of evidence-based practices in online learning: a meta-analysis and review of online learning studies. Retrieved from http://www2.ed.gov/rschstat/eval/tech/evidence-based-practices/finalreport.pdf.

(C) Copyright of Journal of Youth Development Bridging Research and Practice. Content may not be copied or emailed to multiple sites or posted to a listserv without copyright holder's express written permission. Contact Editor at: patricia.dawson@oregonstate.edu for details. However, users may print, download or email articles for individual use.

ISSN 2325-4009 (Print); ISSN 2325-4017 (Online) 